\title{
Rearing of Microplitis mediator (Hymenoptera: Braconidae) and its host Mamestra brassicae (Lepidoptera: Noctuidae)
}

\author{
Elodie BELZ $Z^{1,2}$, CÉline E. GÉNEAU ${ }^{1,3}$, Moritz FÜRST ${ }^{1,4}$, Oliver BALMER ${ }^{1,5, *}$, Pius ANDERMATT ${ }^{6}$, \\ LUKAS PFIFFNER ${ }^{1}$, LÉON WESTERD ${ }^{7}$ and HENRYK LUKA ${ }^{1,8}$
}

\begin{abstract}
${ }^{1}$ Department of Plant Protection and Biodiversity, Research Institute of Organic Agriculture (FiBL), Ackerstrasse 21, Postfach 219, 5070 Frick, Switzerland; e-mails: oliver.balmer@unibas.ch; oliver.balmer@aya.yale.edu; elodie.belz@live.fr; celine.geneau@gmail.com; moritz.fuerst@fibl.org; henryk.luka@fibl.org

${ }^{2}$ Zoological Institute, Department of Environmental Sciences, Faculty of Science, University of Basel, Vesalgasse 1, 4051 Basel, Switzerland

${ }^{3}$ Centre for Sustainable Agriculture, Lancaster Environment Centre, Lancaster University, 10 Lancaster LAI 4YQ, UK

${ }^{4}$ Fachmaturitätsschule (FMS) Basel-Stadt, Engelgasse 120, 4052 Basel, Switzerland

${ }^{5}$ Swiss Tropical and Public Health Institute (Swiss TPH), Socinstrasse 57, 4051 Basel, Switzerland

${ }^{6}$ Technical Insect Rearing, Syngenta Crop. Protection Münchwilen AG, Breitenloh 5, 4333 Münchwilen, Switzerland

${ }^{7}$ Laboratory of Entomology, Wageningen University, P.O. Box 8031, 6700 EH Wageningen, The Netherlands
\end{abstract}

${ }^{8}$ Department of Environmental Sciences, NLU-Biogeography, University of Basel, St. Johanns-Vorstadt 10, 4056 Basel, Switzerland

Key words. Hymenoptera, Braconidae, Microplitis mediator, Lepidoptera, Noctuidae, Mamestra brassicae, pest, parasitoid, cabbage moth, protocol

\begin{abstract}
Establishing continuous and reliable colonies of pest-parasitoid systems in the laboratory is an essential requirement for carrying out manipulative experiments on biological control. Here we describe in detail the rearing protocols that we optimized for the efficient rearing of the cabbage moth Mamestra brassicae and its key parasitoid Microplitis mediator.
\end{abstract}

\section{INTRODUCTION}

The cabbage moth Mamestra brassicae (Linnaeus, 1758) (Lepidoptera: Noctuidae) is widely distributed throughout Asia and Europe (Johansen, 1997) and one of the major pests of cabbage in Europe (Finch \& Thompson, 1992). Its polyphagous larvae feed mainly on cabbage (Brassica oleracea L., Brassicaceae) and other plants in the genus Brassica, but also on tomato, beet, onion and some flowers and forest trees (Hill, 1987). The larvae of M. brassicae are attacked by braconid wasps (Johansen, 1997), among which the solitary larval parasitoid, Microplitis mediator (Haliday, 1834) (Hymenoptera: Braconidae), is the most important (Bianchi et al., 2005; Lauro et al., 2005). It is a generalist endoparasitoid that is reported from approximately 40 different noctuid hosts (Mir Khan, 1999).

Establishing continuous and reliable colonies of these two species in the laboratory is important for biological control purposes. Firstly, a regular supply of $M$. brassicae and M. mediator is necessary to conduct experiments on these species in order to determine how to promote $M$. mediator and so reduce the abundance of M. brassicae (Pfiffner et al., 2003; Bianchi et al., 2005; Lauro et al., 2005; Luo et al., 2010; Géneau et al., 2012; Belz et al., 2013; Géneau et al., 2013). Secondly, mass rearing of M. mediator is a prerequisite for inundative releases. There are earlier reports on how to rear M. brassicae (Agui et al., 1975; Hattori \& Atsusawa, 1980; Browning \& Oatman, 1985), M. mediator (Li et al. 2006) and other species of Microplitis (Powell \& Hartley, 1987; Herard et al., 1988; Greany et al., 1989), which have helped us develop rearing protocols for these species. Here, we present the details of the rearing protocols we have adapted and optimized for our needs and hope other researchers will benefit from our experience.

\section{PROTOCOL FOR REARING MAMESTRA BRASSICAE}

The laboratory colony of M. brassicae at FiBL (Frick, Switzerland) was established using field-collected larvae to which annually 200 new field-collected larvae were added in order to reduce the incidence of the adverse effects due to inbreeding. The colony was kept in a climate chamber at $23 \pm 2^{\circ} \mathrm{C}, 60 \pm 10 \%$ r.h. and under a $16 \mathrm{~L}: 8 \mathrm{D}$ photoperiod.

Freshly emerged adults of both sexes of $M$. brassicae were transferred into a transparent acrylic "egg-laying cylinder" (24 $\mathrm{cm}$ in diameter and $23.5 \mathrm{~cm}$ heigh) (Fig. 1). The bottom of this cylinder consisted of a cardboard disc fixed to the cylinder by an L-shaped aluminium ring ( $1 \mathrm{~cm}$ width, $1 \mathrm{~cm}$ height) that fits around the cylinder. The cardboard disc was covered by a plastic sheet and paper towels to ensure impermeability and absorption of excess humidity. The top of the cylinder was covered by a sheet of green paper fixed by another aluminium ring. The moths laid eggs on two green paper sheets $(A 3,29.7 \mathrm{~cm} \times 42 \mathrm{~cm})$ that covered the inside of the cylinder. The moths were provided with honey and water via rolled up filter papers $(9 \mathrm{~cm}$ diameter, Macherey-Nagel GmbH \& Co. KG, Düren, Germany) inserted through $1 \mathrm{~cm}$ holes in the lid of a Petri dish $(8 \mathrm{~cm}$ in diameter and $3 \mathrm{~cm}$ deep) filled with water. By means of a brush a drop of honey was placed on the top of each rolled up filter paper. Two Petri dishes with five filter papers each were placed in each cylinder.

\footnotetext{
* Corresponding author.
} 
Mamestra brassicae

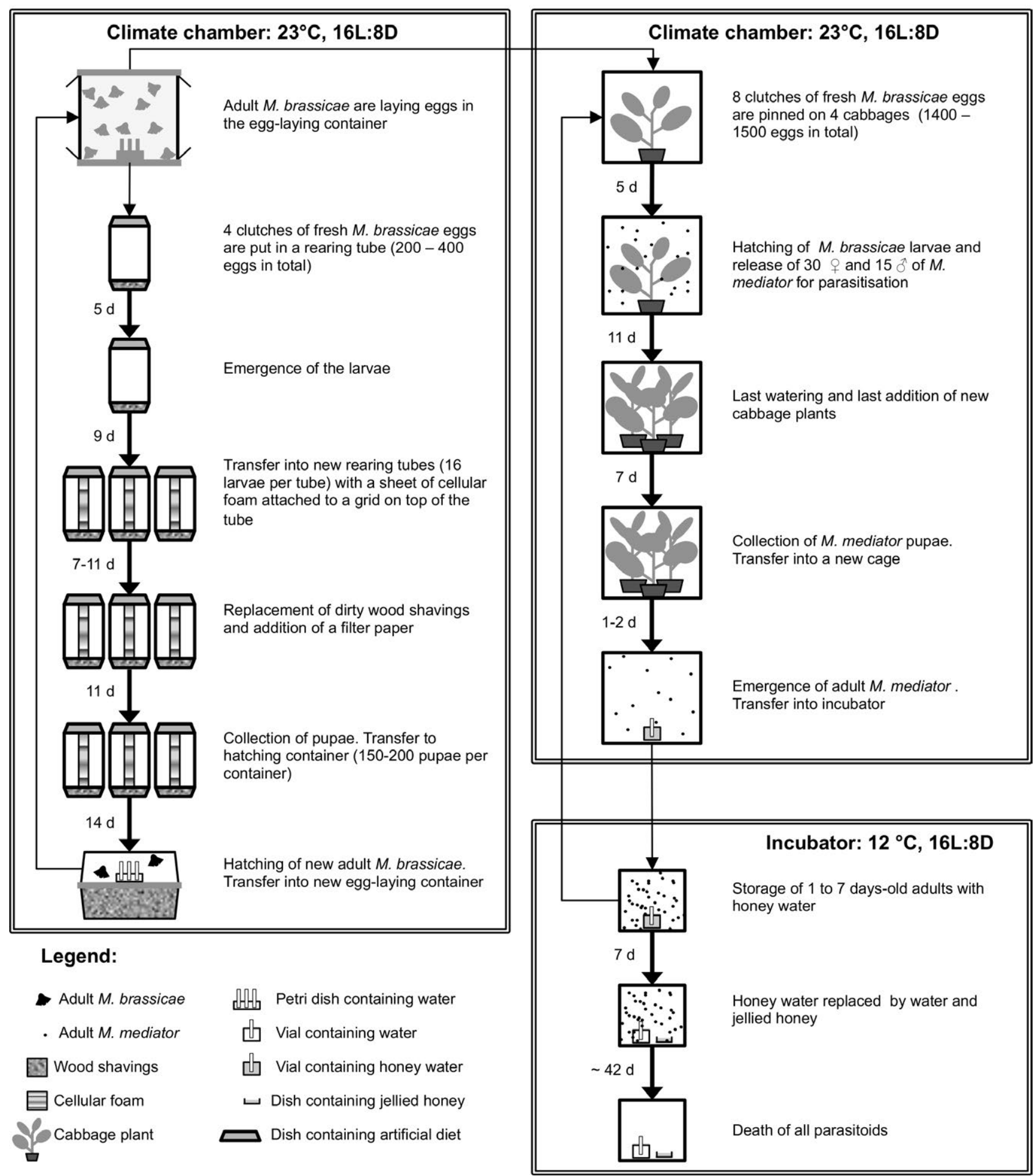

Fig. 1. Chronological steps in the rearing of Mamestra brassicae (left) and Microplitis mediator (right). Times in days between each step are indicated on the left of the bold arrows.

The moths were transferred to clean egg-laying cylinders every two to three days.

To start a new generation (i.e. a complete generation from eggs to adult emergence), four clutches of fresh (i.e. less than 24 h-old) M. brassicae eggs with approximately 200 to 400 eggs per clutch collected from the green paper linning the transparent plastic "rearing tubes" (9 $\mathrm{cm}$ in diameter and $17 \mathrm{~cm}$ long) (Fig. 1). The rearing tubes were each placed in a plastic dish $(10 \mathrm{~cm}$ in diameter at the top, $8 \mathrm{~cm}$ in diameter at the bottom and $5 \mathrm{~cm}$ deep)

filled with sterilized wood shavings that absorbed excess humidity and faeces. The larvae were fed an artificial diet (Beet Armyworm Diet, Bio Serv, Frenchtown, NJ, USA), supplemented with $0.2 \%$ Aurofac 100 (Selectchemie, Zürich, Switzerland) placed in a second inverted plastic dish on the top of the rearing tube. Placing the food at the top prevented it from being contaminated. Tubes and dishes were sealed with Parafilm (Bemis Company, Inc., Neenah, WI, U.S.A.) to prevent the larvae from escaping. 
Larvae of M. brassicae hatched four to six days after they were laid. Fourteen days after egg laying, batches of 16 larvae were transferred to new rearing tubes to reduce the competition between the larvae (Fig. 1). These tubes do not need to be sealed as the larvae are now too big to escape. A metal grid $(4 \mathrm{~cm} \times 8 \mathrm{~cm})$ was placed across the opening of the rearing tube, under the food dish, and a strip of cellular foam (approximately $23 \mathrm{~cm} \times 5 \mathrm{~cm}$ ) fixed to the grid with staples so that it touched the wood shavings. The cellular foam helps the larvae climb onto the grid to access the diet, which is particularly important from instar III onwards when the larvae are too heavy to climb up the walls of the rearing tubes. The number of rearing tubes depends on the number of larvae needed for experiments. Twenty-one to 25 days after the eggs were laid the dirty top layer of wood shavings was removed. A piece of filter paper with an approximately $45^{\circ}$ triangle cut out of its centre was placed on the wood shavings and approximately $1 \mathrm{~cm}$ layer of fresh wood shavings spread over it. This allows the larvae to dig into the wood shavings under the filter paper for pupation while being protected from the faeces of the larvae that are still feeding.

The pupae were collected from all of the rearing tubes 32 to 36 days after the eggs were laid and placed in an "eclosion container" (24 cm in diameter and $8 \mathrm{~cm}$ high) (Fig. 1), which consisted of two transparent plastic dishes held together by a two-sided Tshaped aluminium ring $(24.5 \mathrm{~cm}$ in diameter $\times 1 \mathrm{~cm}$ wide $\times 2 \mathrm{~cm}$ deep). The top dish served as the lid. The pupae were placed in the bottom plastic dish in 3 layers of 50 to 60 pupae each separated by and covered by a layer of wood shavings, so that all the pupae are properly buried. The larvae that did not pupate were left in the rearing tubes for one more week. At FiBL, the percentage pupation was approximately $73 \%$. Adult moths started to eclose approximately 14 days after pupation. The eclosion success of $M$. brassicae pupae was approximately $72 \%$. Newly eclosed moths were transferred to an egg-laying cylinder. Water (without honey) was provided as described above and renewed as needed. The eclosion containers were checked and cleaned with a paper towel to remove singly laid eggs and then stood in $2 \mathrm{~cm}$ of soapy water in order to prevent mites entering the containers.

The pupae of $M$. brassicae can be stored at $4^{\circ} \mathrm{C}$ for many months in either sterilized soil or wood shavings covered with filter paper. When wood shavings are used the container must be sealed with Parafilm to avoid desiccation. After four to six months of storage, $70.03 \pm 3.06 \%$ of the pupae were still alive, i.e. exhibited some abdominal movements when gently squeezed with insect forceps ( $n=17$ generations and a total of 2005 pupae). Moths emerged from approximately $85 \%$ of the pupae.

All materials were washed in soapy water and sprayed with $70 \%$ ethanol after use, with the exception of the egg-laying cylinders, which were not sprayed with ethanol as it causes the acrylic glass of the cylinders to crack. Most of the time was spent transferring larvae to new rearing tubes, adding filter paper after removal of the dirty wood shavings and collecting pupae. Processing 20 new rearing tubes per week adds approximately 45, 60 and $60 \mathrm{~min}$, respectively, to the work load.

\section{REARING MICROPLITIS MEDIATOR ON HOSTS FED ON CABBAGE}

Parasitoids were reared on $M$. brassicae larvae fed on white cabbage Brassica oleracea L. var. capitata L. (Brassicaceae) in a climate chamber at $23 \pm 1{ }^{\circ} \mathrm{C}, 50 \pm 15 \%$ r.h. and under a $16 \mathrm{~L}: 8 \mathrm{D}$ photoperiod. Cabbage plants were grown from seeds at $21 \pm 2^{\circ} \mathrm{C}$, $50 \pm 15 \%$ r.h. and under a $12 \mathrm{~L}: 12 \mathrm{D}$ photoperiod in trays $(33 \mathrm{~cm}$ $\times 51 \mathrm{~cm}$ ) of soil (Einheitserde Classic, Gebrüder Patzer $\mathrm{GmbH}$ \& Co.KG, Germany) fertilized with $3 \mathrm{~g} / \mathrm{L}$ of Tardit $3 \mathrm{M}$ (Hauert HBG Dünger AG, Switzerland). After three to four weeks the

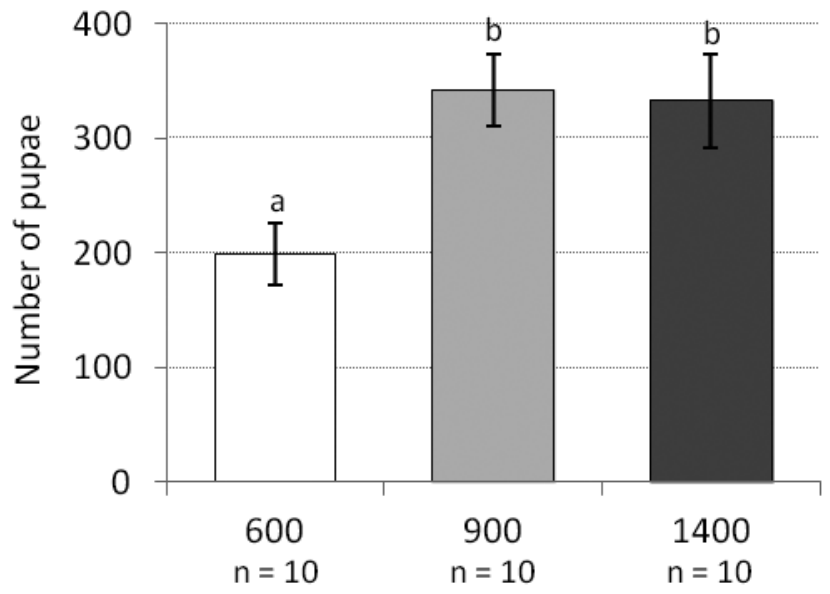

Fig. 2. Comparison of the mean $( \pm \mathrm{SE})$ number of pupae of Microplitis mediator collected from cages in which the initial numbers of Mamestra brassicae eggs were either 600, 900 or 1400. Columns with different letters differ significantly from each other (linear model, $\mathrm{p}<0.01$ ).

plants were transplanted into pots $(12 \mathrm{~cm}$ diameter, $10 \mathrm{~cm}$ height $)$ containing soil and the same amount of fertilizer and transferred to a greenhouse. Three to four weeks after transplantation the then 11 to 15-leaf stage plants were used as food for M. brassicae larvae. The youngest larvae were placed on the youngest cabbage plants with the thinnest leaves.

To produce a cohort of parasitoids eight recently laid clutches of eggs of M. brassicae were pinned onto the underside of the leaves of four cabbage plants, which in our case amounted to approximately 1400 eggs per cage. The plant pots were placed in a plastic dish, so that they could easily be watered from below, and then in an insect rearing cage $(47.5 \mathrm{~cm} \times 47.5 \mathrm{~cm} \times 47.5 \mathrm{~cm}$, BugDorm-44545F, MegaView Science Co., Taichung, Taiwan). Any water spilt was absorbed by paper placed under the plastic dish. The Mamestra brassicae larvae hatched after five days. Approximately 30 females and 15 males of M. mediator (two-day to one week-old) were added to each cage on the day the M. brassicae larvae hatched, or one day before because this parasitoid parasitizes the youngest larvae with the highest efficiency (Géneau et al., 2012). A paper towel spread with honey was suspended from the top of the cage and sprayed with water to ensure that the parasitoids survived long enough to parasitize the larvae. Three days later the paper towel was removed.

Between 7 and 10 fresh cabbage plants were added as needed over a period of 11 days following parasitization, which is when the parasitoid larvae were about to pupate. Cabbage plants were only watered for 11 days after parasitization in order to prevent the material in the cage becoming very wet when the parasitoids pupated.

The pupae of M. mediator were collected by hand or with the aid of insect forceps 18 days after parasitisation, when the entire cage was checked systematically. They were carefully removed from the substrate and transferred to an open Petri dish in a rearing cage in which there was an ad libitum supply of honey water. The honey water was in a small vial $(4.5 \mathrm{~cm}$ diameter, $4 \mathrm{~cm}$ height) and prepared by mixing approximately three grams of honey with $25 \mathrm{ml}$ of water. The vials were replaced every two to three days to prevent fermentation and mould developing. A rolled up filter paper was inserted through a $0.5 \mathrm{~cm}$ hole pierced in the lid of each vial.

We conducted an experiment to determine whether the rearing efficiency (i.e. the number of $M$. mediator pupae collected per $M$. brassicae egg exposed) could be increased by decreasing the 
number of M. brassicae eggs in each cage. We placed either 600, 900 or 1400 eggs of M. brassicae in each cage and determined the number of pupae of M. mediator that developed ( $\mathrm{n}=10$ cages for each initial number of eggs). The mean numbers $( \pm \mathrm{SE})$ of pupae that developed were $199.2 \pm 27.3$ in the 600 egg treatment, $342.4 \pm 31.7$ in the 900 egg treatment and $332.8 \pm 40.6$ in the 1400 egg treatment (Fig. 2), which in terms of percentage parasitism and pupation are $33.2 \pm 4.6 \%, 38.1 \pm 3.5 \%$, and $22.9 \pm 2.6 \%$, respectively, in the three treatments. Comparison of the numbers of pupae collected in the different treatments using linear model in R 2.13.2 (R Development Core Team, 2005) revealed that there were significant differences between the 600 egg treatment and the two other treatments ( 900 eggs: $\mathrm{t}=3.006, \mathrm{df}=27, \mathrm{p}=0.0057$ and 1400 eggs: $t=2.805, d f=27, p=0.0092)$, but not between the 900 and 1400 egg treatments $(t=0.202, d f=27, p=0.8418)$ (Fig. 2), indicating that the highest efficiency measured in terms of number of pupae collected per egg exposed was achieved with 900 eggs.

At $23 \pm 1^{\circ} \mathrm{C}$, adult parasitoids started to emerge from the pupae one to two days after they were collected. At FiBL, the mean percentage emergence was approximately $96 \%$ and mean percentage that were female was $50 \%$. The pupae of $M$. mediator can be stored for a few days at 10 to $12^{\circ} \mathrm{C}$ to delay emergence of adults. Newly emerged parasitoids were collected regularly using a Dcell vacuum insect aspirator (MX-991/U, Hausherr's Machine Works, Tom Rivers, NJ, U.S.A.), placed in a small rearing cage $(24.5 \times 24.5 \times 24.5$, Bugdorm-42222F, MegaView Science Co., Taichung, Taiwan) in an incubator at $12 \pm 1^{\circ} \mathrm{C}, 60 \pm 2 \%$ r.h. under a $16 \mathrm{~L}: 8 \mathrm{D}$ to decrease their metabolic activity and increase their lifespan. During the first week, they were provided with honey water ad libitum to ensure that they were well fed before being used in parasitization experiments. The remaining parasitoids were kept as stock in an incubator until they died. They were provided with water (using the same kind of vial and filter paper as in the honey water treatment) and 3 to $5 \mathrm{ml}$ of jellied honey in a small plastic dish. Parasitoids are less able to ingest jellied honey, but it has the advantage of lasting for weeks. Jellied honey is prepared by mixing $3 \mathrm{~g}$ of white gelatine (Dr Oetker AG/SA, Obergösgen, Switzerland) with $100 \mathrm{ml}$ of distilled water and then slightly heating this solution and adding $200 \mathrm{~g}$ of honey. This mixture is poured into syringes (to facilitate dispensing) and kept in a refrigerator at $4^{\circ} \mathrm{C}$ until required.

The transplanting of cabbage plants and the collection of pupae took up most of the time. At a production rate of 15 cabbages per week the transplanting of the cabbage plants took about one hour. It took approximately $1.5 \mathrm{~h}$ per cage to collect the pupae.

\section{REARING OF MICROPLITIS MEDIATOR ON HOSTS REARED ON ARTIFICIAL DIET}

We developed a protocol for rearing M. mediator on M. brassicae fed on the same artificial diet as used to rear M. brassicae. However, rearing them in this way was not continued for very long so it is not possible to judge whether rearing them in this way would affect the behaviour of adult $M$. mediator, as is reported for another parasitoid (Bautista \& Harris, 1997; Gandolfi et al., 2003).

This rearing was done under the same environmentsl conditions as the rearing on cabbage. A recently laid clutch of $30 \mathrm{M}$. brassicae eggs was put in a small "parasitization vial" $(4.5 \mathrm{~cm}$ in diameter and $4 \mathrm{~cm}$ long) with a $1.5 \mathrm{~cm}$ diameter hole in the lid to facilitate air exchange. Another $1.5 \mathrm{~cm}$ diameter hole pierced in the side of the parasitization vial had inserted and glued into it a little flexible plastic tube $(1.5 \mathrm{~cm}$ in diameter and $1.5 \mathrm{~cm}$ long), so that $1 \mathrm{~cm}$ of the tube protruded from the side of the vial. The opening of the tube was closed by inserting an Eppendorf tube (1 $\mathrm{cm}$ in diameter and $4 \mathrm{~cm}$ long) into it. A layer of artificial diet (approx. $1 \mathrm{~cm}$ deep) was poured into the parasitization vial, which was then inverted so that the artificial diet was at the top of the vial. A piece of paper towelling was placed between the vial and the lid to prevent the larvae from escaping and absorb excess humidity.

The eggs of $M$. brassicae in the parasitization vials took five days to hatch after which four two-day to one-week old adults of $M$. mediator (two females and two males) were introduced into each vial through the plastic tube inserted in the side of each vial. The opening of the tube was closed after all the parasitoids entered the vial. Small vials were used to ensure that the female parasitoids were able to find all the larvae and maximize the percentage parasitism, a technique that was developed in a previous study (Géneau et al., 2012).

Two days after parasitization, the larvae of $M$. brassicae were transferred to a modified rearing tube (see Mamestra brassicae rearing protocol), the bottom of which consisted of paper toweling fixed to the tube by a rubber band instead of a plastic dish filled with wood shavings. Wood shavings are not needed because the larvae of $M$. brassicae do not pupate but continue to develop until the parasitoid larvae pupate 16 days after transfer to the rearing tube. The pupae were collected as described when they were reared on cabbage. The mean number of pupae collected per tube was $7.7 \pm 1.6$, which represents a percentage parasitism and pupation of M. mediator of $25.5 \pm 5.4 \%$.

\section{DISCUSSION}

The percentage of eggs of M. brassicae that hatched and of the larvae that pupated and gave rise to adults were all very high. The use of the rearing tubes made it possible to produce large numbers of individuals in a relatively limited space. By limiting the contact the larvae have with faeces decreased the risk of their being adversely affected by the mould that developed on the faeces and separating the larvae into several rearing tubes also limited the potential spread of diseases as contaminated tubes were put to one side or discarded. The use of an artificial diet resulted in a considerable saving in time as fifth instar larvae consume large amounts of food. A problem with this approach is that it could influence the behaviour of adult moths as the choice of an oviposition site by Spodoptera littoralis (Boisduval, 1833) (Lepidoptera: Noctuidae) is influenced by the plant on which the larvae developed (Shikano \& Isman, 2009) and the host searching ability of adult females of some parasitoid species is affected when they are reared on an artificial diet (Bautista \& Harris, 1997; Gandolfi et al., 2003). Thus, it is important to determine whether M. brassicae reared on an artificial diet behave differently if they are to be used in behavioural studies. In our case they were reared on an artificial diet because we only needed eggs for exposing to natural enemies in the field (Balmer et al., 2013) or adults for determining their fecundity when fed on different wild plants (Géneau et al., 2012).

The M. mediator were reared on hosts fed on cabbage because we used the adult parasitoids for behavioural studies (Belz et al., 2013). But as it is possible to rear parasitoids in hosts fed an artificial diet this could be a good starting point for developing a mass rearing technique for $M$. mediator. If the aim is to use M. mediator as a biological control agent in the field, its behaviour when reared on $M$. brassicae fed on artificial diet should be critically investigated, because any decrease in the host searching ability of female parasitoids is undesirable.

Greater efficiency in rearing the parasitoid than M. brassicae is more difficult to achieve. The percentages that pupated were quite low, whatever the diet or protocol used. Our results indicate that the use of intermediate numbers of eggs of $M$. brassicae results 
in a higher percentage of larvae pupating. The low percentage pupation recorded for M. mediator in the treatment in which 1400 M. brassicae eggs were exposed to parasitization by 30 females of $M$. mediator might indicate that the total number of larvae they could parasitize was limited by the number (900) of eggs they could lay or the mortality of the $M$. brassicae larvae developing from the 1400 eggs was higher. The percentage parasitism might be increased by introducing more female $M$. mediator into the cages, but this could also lead to increased intra-specific competition between females (Greenberg et al., 1995), increased mortality of the $M$. brassicae larvae and decreased emergence of adult parasitoids (Shepard \& Gale, 1977). Despite the low percentage pupation recorded for $M$. mediator the number of pupae collected at the end of each generation was always sufficient for our experiments.

In conclusion, our rearing of $M$. brassicae and $M$. mediator proved to be reliable over the course of many years and easy to adjust so that the production met our needs by changing the number of generations initiated each week.

ACKNOWLEDGEMENTS. We thank R. Gols (Wageningen, Netherlands) for providing the pupae of $M$. mediator we used to start our rearing of this species and O. Kindler (Syngenta, Stein, Switzerland) for providing the artificial diet used in this study. This work was supported by the Bristol-Foundation, the Ministry for Environment, the Ernst Göhner Foundation, the ParrotiaFoundation, the Spendenstiftung Bank Vontobel, the Werner Steiger Foundation, the Foundation for International Conservation of Plant Diversity, the Singenberg Foundation and Schöni Swissfresh AG.

\section{REFERENCES}

Agui N., Ogura N. \& Okawara M. 1975: Rearing of the cabbage armyworm, Mamestra brassicae L. (Lepidoptera: Noctuidae) and some lepidopterous larvae on artificial diets. - Jap. J. Appl. Entomol. Zool. 19: 91-96.

Balmer O., Pfiffner L., Schied J., Willareth M., Leimgruber A., Luka H. \& Traugott M. 2013: Noncrop flowering plants restore top-down herbivore control in agricultural fields. - Ecol. Evol. 3: 2634-2646.

BAUTISTA R.C. \& HARRIS E.J. 1997: Effect of insectary rearing on host preference and oviposition behavior of the fruit fly parasitoid Diachasmimorpha longicaudata. - Entomol. Exp. Appl. 83: $213-218$.

Belz E., Kolliker M. \& Balmer O. 2013: Olfactory attractiveness of flowering plants to the parasitoid Microplitis mediator: potential implications for biological control. - BioControl 58: $163-173$

Bianchi F.J.J.A., Van Wingerden W.K.R.E., Griffioen A.J., Van der Veen M., Van der Straten M.J.J., Wegman R.M.A. \& Meeuwsen H.A.M. 2005: Landscape factors affecting the control of Mamestra brassicae by natural enemies in Brussels sprout. - Agricult. Ecosyst. Environ. 107: 145-150.

Browning H.W. \& OATMAN E.R. 1985: Reproductive biology of Microplitis brassicae (Hymenoptera, Braconidae), parasite of the cabbage looper, Trichoplusia ni (Lepidoptera, Noctuidae). - Ann. Entomol. Soc. Am. 78: 369-372.

Finch S. \& Thompson A.R. 1992: Pests of cruciferous crops. In Mc Kinlay R.G. (ed.): Vegetable Crop Pests. Mac Millan, London, pp. 87-138.

Gandolfi M., Mattiacci L. \& Dorn S. 2003: Mechanisms of behavioral alterations of parasitoids reared in artificial systems. - J. Chem. Ecol. 29: 1871-1887.

Géneau C.E., Wäckers F.L., Luka H., Daniel C. \& Balmer O. 2012: Selective flowers to enhance biological control of cabbage pests by parasitoids. - Basic Appl. Ecol. 13: 85-93.
Géneau C.E., Wäckers F.L., LuKa H. \& BaLmer O. 2013: Effects of extrafloral and floral nectar of Centaurea cyanus on the parasitoid wasp Microplitis mediator: olfactory attractiveness and parasitization rates. - Biol. Contr. 66: 16-20.

Greany P.D., Ferkovich S.M. \& Clark W.R. 1989: Progress towards development of an artificial diet and an in vitro rearing system for Microplitis croceipes. - Southw. Entomol. (Suppl.) 12: 89-94.

Greenberg S.M., Morales-Ramos J.A., King E.G., Summy K.R., \& RoJAS M.G. 1995: Biological basis for mass propagation of Catolaccus grandis (Hymenoptera: Pteromalidae): effects of parasitoid densities and host-parasitoid ratios. - Environ. Entomol. 24: 1322-1327.

Hattori M. \& Atsusawa S. 1980: Mass rearing of the cabbage armyworm, Mamestra brassicae Linné and the common armyworm, Mythimna separata Walker (Lepidoptera, Noctuidae) on a simple artificial diet. - Jap. J. Appl. Entomol. Zool. 24: 36-38.

Herard F., Keller M.A. \& Lewis W.J. 1988: Rearing Microplitis demolitor Wilkinson (Hymenoptera, Braconidae) in the laboratory for use in studies of semiochemical mediated searching behavior. - J. Entomol. Sci. 23: 105-111.

HiLl D.A. 1987: Agricultural Insect Pests at Temperate Regions and their Control. Cambridge University Press, Cambridge, $659 \mathrm{pp}$.

JohANSEN N.S. 1997: Mortality of eggs, larvae and pupae and larval dispersal of the cabbage moth, Mamestra brassicae, in white cabbage in south-eastern Norway. - Entomol. Exp. Appl. 83: 347-360.

Lauro N., Kuhlmann U., Mason P.G. \& Holliday N.J. 2005: Interaction of a solitary larval endoparasitoid, Microplitis mediator, with its host, Mamestra brassicae: host acceptance and host suitability. — J. Appl. Entomol. 129: 567-573.

Li J., Yan F., Coudron T.A., Pan W., Zhang X., Liu X. \& Zhang Q. 2006: Field release of the parasitoid Microplitis mediator (Hymenoptera: Braconidae) for control of Helicoverpa armigera (Lepidoptera: Noctuidae) in cotton fields in northwestern China's Xinjiang province. - Environ. Entomol. 35: 694-699.

Luo S., Li J., Liu X., Lu Z., Pan W., Zhang Q. \& Zhao Z. 2010: Effects of six sugars on the longevity, fecundity and nutrient reserves of Microplitis mediator. - Biol. Contr. 52: 51-57.

Mir Khan S. 1999: Effectiveness of Microplitis mediator (Hym.: Braconidae) against its hosts Agrotis segetum and A. ipsilon (Lepidoptera: Noctuidae). - Pakist. J. Biol. Sci. 2: 344-346.

Pfiffner L., Merkelbach L. \& Luka H. 2003: Do sown wildflower strips enhance the parasitism of lepidopteran pests in cabbage crops? - IOBC/WPRS Bull. 26: 111-116.

Powell J.E. \& Hartley G.G. 1987: Rearing Microplitis croceipes (Hymenoptera, Braconidae) and other parasitoids of Noctuidae with multicellular host-rearing trays. - J. Econ. Entomol. 80: 968-971.

R Development Core Team 2005: R: A Language and Environment for Statistical Computing. R Development Core Team, Vienna, Austria.

ShePARd M. \& Gale G.T. 1977: Superparasitism of Epilachna varivestis (Col.: Coccinellidae) by Pediobius foveolatus (Hym.: Eulophidae): influence of temperature and parasitoidhost ratio. - Entomophaga 22: 315-321.

SHIKANO I. \& Isman M.B. 2009: A sensitive period for larval gustatory learning influences subsequent oviposition choice by the cabbage looper moth. - Anim. Behav. 77: 247-251.

Received October 30, 2013; revised and accepted January 6, 2014 Prepublished online May 6, 2014 